\title{
Quand une société se transforme. L'évolution de la formation des instituteurs valaisans entre 1950 et 1970
}

\section{Danièle Périsset Bagnoud}

Cette contribution analyse une partie des transformations de l'école publique valaisanne entre 1950 et 1970 du point de vue des politiques cantonales de l'éducation. Les conséquences de l'avènement de la modernité et de la démocratisation des études se font grandement sentir à cette période, notamment parce que le canton avait, jusque-là, fortement contenu son évolution industrielle et maintenu sa tradition rurale politique et sociale. Dans les années '60, les temps changent. Les études se démocratisent, les écoles secondaires se généralisent et les emplois dans le secteur tertiaire attirent les jeunes. Le métier d'instituteur ne fait plus recette; de nouveaux modèles de formation sont inventés qui laissent anticiper entre autre la disparition de l'institut traditionnel de formation des institutrices et des instituteurs qu'est l'Ecole normale.

\section{L'exemple du Valais}

L'extension des systèmes de formation dans les années ' 50 à '70 est, en Occident, impressionnante. La croissance économique de ces décennies-là fait exploser qualitativement et quantitativement les formes et structures des formations proposées jusqu'alors. Le Valais, canton à forte tradition conservatrice catholique rurale, illustre de manière frappante les tensions induites par les transformations sociales et économiques de cette période.

Dans la vallée alpine qui en constitue le territoire, le développement industriel est strictement contenu tout au long du $19^{\mathrm{e}}$ siècle de même qu'au début du $20^{\mathrm{e}}$. Ainsi, au parlement valaisan, les revendications ouvrières sont diabolisées (voir Arlettaz, 1979; Clavien, 1988). Le canton tient à son identité construite autour de la fidélité à l'Eglise de Rome, à la soumission à l'ordre conservateur clérical, au rejet de toute forme de laïcité et d'individualisation des valeurs morales. Dans son discours et dans ses décisions, l'élite politique installée solidement et sans contestation au gouvernement cantonal depuis 1857 rejette dans la mesure du possible toute forme de modernité (voir à propos de la modernité dans les pays catholiques Altermatt, 1994). 
La Seconde Guerre mondiale s'achève lorsque le Valais politique prend acte des transformations induites par le conflit, notamment dans le domaine de l'instruction publique:

A l'époque des grands bouleversements, la société éprouve le besoin de faire l'inventaire de ses valeurs, de les réviser et de donner à l'enseignement une orientation conforme aux aspirations du moment. Une des tendances de l'école, aujourd'hui, est de glisser de l'école-savoir vers une conception plus utilitaire et vers une éducation fonctionnelle. Nous n'irons pas jusqu'à nous faire l'apôtre de ces idées nouvelles, mais nous estimons que le personnel enseignant doit les connaître pour, le cas échéant, en tirer profit et en faire une application judicieuse dans nos écoles (Rapport de gestion du Conseil d'Etat, DIP, 1944, p. 5).

L'essentiel du conflit idéologique et social qui oppose la classe politique dominante valaisanne, conservatrice catholique, aux exigences de l'industrialisation naissante du canton (voir à ce propos Arlettaz, 1976) et du mieux-être économique que la population des campagnes commence enfin à entrevoir se lit dans cette citation. L'après-guerre oblige à réformer l'instruction publique dans le sens des besoins de l'industrialisation croissante et des aspirations de la population; l'idéal politique tendrait plutôt, lui, vers une reproduction des valeurs traditionnelles (soumission, zèle, humilité, piété, dévouement). Aspirations matérielles et industrielles areligieuses contre idéologie catholique traditionnelle: la volonté de concilier les impératifs antinomiques ainsi résumés ne va pas sans susciter diverses contradictions.

La présente contribution analyse la période charnière qui court de la fin des années '40 à 1970 du point de vue des politiques cantonales en matière d'éducation et d'instruction publique. Les débats parlementaires et les réalisations législatives enregistrées dans ce cadre en témoigneront.

\section{Avant 1950: une instruction publique au service de la reproduction d'une société dite traditionnelle}

Au cours du $19^{\mathrm{e}}$ siècle, l'Etat du Valais a pris sous son aile l'école publique valaisanne au terme d'un long combat: ici comme ailleurs, le salut des âmes a dirigé les premières mesures d'instruction du peuple, bien avant que les états nationaux ne s'en préoccupent. L'introduction de la démocratie les pousse à s'arroger le "gouvernement des esprits» (selon l'expression de Nique, 1991), engageant pour cela d'âpres batailles avec le pouvoir ecclésiastique peu enclin à céder son incontestable emprise sur le peuple (voir Criblez, Hofstetter \& Périsset Bagnoud, 2000; Hofstetter, Magnin, Criblez \& Jenzer, 1999; Nique, 1989, 1991). Mais, alors que, le plus souvent, les cléricaux sont définitivement écartés de cette mission éducative, en Valais, les intérêts séculiers et religieux pactisent et se répartissent les rôles dans les textes de loi. Dans les faits, cette répartition n’interdit nul- 
lement que chacun intervienne dans le domaine qui ne lui est pas officiellement dévolu, les uns servant le pouvoir des autres et réciproquement (voir Périsset $\mathrm{Ba}-$ gnoud, 2000).

Le pouvoir politique, qu'il soit en mains libérales, radicales ou détenu par des conservateurs plus ou moins ultramontains, souhaite explicitement reproduire un système qui se dit démocratique. Dans ce système, chacun occupe la place que la providence lui a attribuée de naissance: aux élus l'instruction, le pouvoir et la richesse; au peuple le dur et ingrat labeur de la maigre terre des montagnes où trop d'instruction nuit et où la consolation naît de la promesse des bienfaits divins. La référence à la tradition apparaît alors dans les discours et intentions politiques, les vertus qui l'accompagnent sont érigées en valeurs nationales. Elle sert de rempart contre l'ennemi athée et radical devenu, à la fin du $19^{\mathrm{e}}$ siècle, industriel et socialiste. Les Ecoles normales sont créées dans ce contexte social mouvant afin de former des maîtres adéquats. Les besoins cantonaux en matière d'instruction s'accroissent, notamment afin de répondre au défi posé par les examens de recrues organisés par la Confédération (voir Lustenberger, 1997; Métrailler, 1978). La durée des Ecoles normales évolue alors rapidement, passant de quelques semaines à leur création en 1846 à trois années de dix mois en 1904. La mission qui les fonde ne subit cependant aucun changement: former des instituteurs et des institutrices tout à la fois pieux et zélés, instruits sans excès, éduqués à tenir avec dignité leur rôle particulier tout en étant maintenus dans leur modeste condition rurale d'origine (voir Périsset $\mathrm{Ba}$ gnoud, 2000).

Au $19^{\mathrm{c}}$ siècle et dans le premier $20^{\mathrm{c}}$, la durée de la scolarisation annuelle des élèves des écoles valaisannes se résume à celle de l'école primaire, soit à quelques mois par année - rarement au-delà de six - même si quelques voix éclairées cherchent à l'allonger. Les trois collèges du canton, réservés à la future élite cléricale, médicale et juridique du pays, dispensent exclusivement un enseignement humaniste classique (Roduit, 1993). L'agriculture est et doit rester la principale source de revenus: le monde politique n'entend pas déroger à ce principe devenu traditionnel parce que servant ses intérêts propres.

La Seconde Guerre mondiale met à mal ces présupposés. Il devient en effet difficile de concilier la tradition de l'agriculture extensive, malgré les progrès concédés en ce domaine, tel l'assainissement de la plaine du Rhône, et les progrès économiques. La population s'intéresse aux emplois générés par l'industrie naissante, moins pénibles, mieux rémunérés. La défense de l'agriculture comme "autre défense nationale» (Crettol, 1951) ne convainc plus le peuple.

Cette évidence inquiète le monde politique; un ajustement de ses objectifs politico-religieux aux réalités et promesses des inévitables progrès économiques, dont le contrôle et la direction sont vitaux, devient impératif. L'enjeu politique et social des décennies qui vont suivre est ainsi clairement lisible dans le propos du Conseil d'Etat rapporté au début de notre contribution. L'immobilisme est désormais impossible, mais trop de transformations mettrait en danger un équi- 
libre politique et social patiemment construit depuis un siècle. Les débats de la loi de 1947 sur l'instruction publique illustrent les tensions que connaît alors le monde politique.

\section{La Loi sur l'instruction publique de 1947: un essai manqué}

Cette année-là, dans le cénacle du Grand Conseil, un débat nourri tente d'analyser la pénurie de personnel enseignant qui frappe alors l'école publique. Le métier de régent fait encore moins recette qu'hier - phénomène récurrent en période d'embellie économique. Les instituteurs, forts de l'instruction dispensée par la modeste Ecole normale, trouvent sans peine d'autres emplois leur permettant d'entretenir honorablement leur famille: s'ils demeurent régents, leur traitement «est insuffisant pour vivre et trop élevé pour mourir» (Bulletin du Grand Conseil, mai 1947, p. 15). Le statut socio-économique de l'instituteur commence à être pris en considération. Les dirigeants politiques sont bien obligés de reconnaître que le temps où la référence à la «vocation» justifiait un traitement dérisoire est passé, sans pour autant accepter de revaloriser réellement ces salaires étriqués.

Au lendemain de la Seconde Guerre, le contexte national encourage le gouvernement valaisan à revoir la législation scolaire. Nombreux sont en effet les cantons suisses qui réforment alors leur scolarité obligatoire et la prolongent en vue de l'améliorer. La loi valaisanne du début du siècle (1907) ne faisait que réorganiser les diverses améliorations apportées à la loi de 1873. Elle est désormais jugée caduque. Bien que ne voulant pas se faire l'apôtre des idées nouvelles, le gouvernement se trouve dans l'obligation de tenir compte des tendances aspirant à un enseignement mieux ajusté aux besoins des industries locales naissantes. Mais, très prudent, il n'innove pas réellement. Ainsi, la prolongation de la scolarité au-delà des six mois effectués jusque-là n'est pas inscrite dans le projet de loi. Quelques communes rurales avaient tenté l'expérience: la pression populaire y a rapidement mis un terme. Les temps ne semblent pas mûrs. Les politiciens ne veulent pas contrarier le peuple des agriculteurs de montagne. D'ailleurs, le gouvernement est peu enclin à anticiper une évolution économique qui n'entre pas dans son projet de maintien d'une société agricole. Seuls sont proposés l'avancement facultatif de l'âge d'entrée dans la scolarité obligatoire, une augmentation des subventions cantonales pour les bâtiments scolaires et un encouragement au dédoublement des classes.

L'image que le gouvernement se fait des conséquences d'un mieux-être économique du peuple lui fait craindre l'abandon des valeurs traditionnelles. Afin de prévenir les éventuelles tentations que la modernité pourrait offrir à la population et pour garantir la pérennité d'un bonheur calé sur la répartition traditionnelle des rôles, il est encore décidé d'obliger toutes les jeunes filles entre 14 et 16 ans à suivre un cours d'enseignement ménager dans leur commune: 
Je dis que ce serait un progrès social énorme de préparer les jeunes filles à leur rôle futur, de préparer le bonheur de leur foyer en leur apprenant l'économie domestique, l'utilisation des produits du sol, l'hygiène, etc. Par conséquent, je suis heureux de voir que ce point de vue est celui de la Haute assemblée (Bulletin du Grand Conseil, 1946, pp. 15-16).

Bref, à la grande colère des quelques députés soucieux de faire évoluer l'école vers les besoins nouveaux (voir le Bulletin du Grand Conseil, nov. 1946, p. 125), l'éducation ménagère des jeunes filles est la seule réelle innovation introduite par la loi de 1947.

La question de l'évolution de l'école primaire resurgit lors des débats qui mettent en cause l'Ecole normale. "Pour former de bons citoyens, il faut de bons maîtres» (Rapport sur les Ecoles normales au DIP, 1944. AEV, 4150-6/M7): la formule est explicite. Le gouvernement place sa confiance dans les Ecoles normales en fonction à présent depuis un siècle. Il ne saurait être question de confier la formation des instituteurs aux collèges à qui il appartient de former les futures élites cantonales. Il faut aux éducateurs des enfants du peuple une instruction modeste, adaptée aux besoins du pays. C'est exactement celle que les Frères de Marie offrent aux jeunes gens et les Sœurs Ursulines aux jeunes filles. La mission confiée aux congrégations religieuses demeure donc inchangée. Seule la prolongation de la formation en Ecole normale est envisagée.

Malgré les progrès sensibles de l'industrialisation du pays, en 1947, le gouvernement a réussi à maintenir l'école publique en l'état, concédant quelques améliorations qui renforcent, de fait, ses propres buts politiques. Les tenants du progrès et d'une entrée de la société valaisanne dans la modernité n'en démordent cependant pas: l'école publique doit désormais tenir compte des besoins nouveaux en instruction, besoins issus du développement des secteurs économiques secondaire et tertiaire. L'évolution des autres cantons suisses et l'évidence de l'évolution de la structure de l'emploi en Valais justifient leur obstination.

\section{Ajuster la formation des instituteurs aux besoins sociaux}

De fait, et malgré la pression traditionnelle toujours présente au niveau du discours politique, la demande sociale en matière d'instruction se transforme rapidement dans les années ' 50 . Lui faisant écho en 1955, une motion réclame une réforme conséquente de l'Ecole normale au nom du bien de l'école publique (voir AEV, 4150-7/67; -7/1b; -7/3d).

Son auteur, le député Joseph Moulin, souhaite une transformation de l'école publique conforme à l'évolution économique du canton. L'importance de la personnalité du maître et son impact sur l'éducation des élèves sont invoqués dans son argumentaire. L'Ecole normale, celle des instituteurs en particulier, est directement mise en cause: il lui faut se moderniser dans une juste mesure afin de per- 
durer et de conserver son influence sur le peuple. Le parlementaire réclame «un projet prévoyant une sélection des candidats qui présentent les meilleurs indices de dons personnels pour l'éducation» (Motion, AEV, 4510-7/67). La formation de ces éducateurs est également à revoir. Il faut tenir compte des exigences économiques contemporaines afin de mieux maîtriser les transformations sociales en cours: une augmentation de la qualité de la formation et une réforme de ses contenus sont souhaitées. Comparée à l'immobilisme qui entoure la loi sur l'instruction publique de 1947, la rapidité avec laquelle le Département de l'instruction publique (DIP) prend en compte la motion Moulin, et agit, indique l'intérêt porté à la question de la modernité. Le souci de conserver la haute main sur la formation des mentalités, malgré l'inéluctable évolution sociale, est patent.

Pour former de bons citoyens, il faut former de bons maîtres: l'Ecole normale demeure, en ces années de changement social structurel, l'instrument éducatif dont le gouvernement a besoin. Il faut donc soigner particulièrement l'institution, en fonction d'objectifs précis mais traditionnels. Selon le directeur de l'Ecole normale des jeunes gens, les instituteurs ne sont-ils pas «des esprits pratiques qui pèseraient sur le niveau des études classiques et qui deviennent quand même, à force de travail et de dévouement, de bons maîtres»? Ne sont-ils pas issus de milieux économiquement faibles ne pouvant s'offrir de longues études hors de leur foyer (Rapport sur la réforme de l'Ecole normale présenté par le directeur Lorétan. AEV, 4150-7/1b)? Le pays ne rencontre-t-il pas maints avantages à laisser son personnel enseignant être formé par des religieux (Rapport sur les avantages d'ordre moral [...] par le directeur Lorétan. AEV, 4150-7/3d)? Les objectifs sociaux du gouvernement entrent, alors comme à la fin du $19^{\mathrm{e}}$ siècle, en résonances avec le charisme et les convictions des congrégations religieuses en charge des Ecoles normales. La convergence d'idéal (catholique conservateur) et la concomitance des intérêts gouvernementaux et religieux fondent l'évolution de la structure de la formation des instituteurs, sans transformer l'essence de sa mission.

Des mesures sont donc immédiatement prises. Ainsi, la procédure de sélection des candidats est corrigée. Désormais, un examen psychotechnique ${ }^{1}$ complétera l'examen de connaissances générales scolaires, et les connaissances générales requises iront au-delà des savoirs proposés lors de la scolarité primaire. Deux cycles d'études restructureront la formation professionnelle et générale des futurs instituteurs. ${ }^{2}$ Ainsi, les deux premières années seront consacrées à la formation générale tandis que, pendant les deux dernières, un accent particulier sera mis sur les branches professionnelles (déjà présentes dans le plan d'étude depuis 1935 au moins: psychologie, pédagogie, sociologie selon la doctrine sociale de l'Eglise catholique, enseignement pratique). Une année d'orientation est ensuite introduite en 1961, année qui comptera bientôt comme la cinquième année d'études à l'Ecole normale. Cette année supplémentaire parachève le dispositif de sélection des futurs instituteurs: les candidats n'ayant pas obtenu le minimum de points nécessaires à leur promotion ou n'ayant pas démontré les 
qualités requises pour un futur éducateur devront quitter l'Ecole et réorienter leur formation professionnelle à l'issue de cette année probatoire. ${ }^{3}$

Le Conseil d'Etat estime avoir ainsi modernisé l'institution de formation des maîtres et, par conséquent, l'école publique, tout en préservant leur orientation catholique et en contenant d'éventuels excès modernistes.

\section{La transformation de la demande d'instruction}

La motion Moulin dont est issue la réforme de l'Ecole normale n'est pas isolée; plusieurs autres motions et postulats sont déposés au Parlement entre 1950 et 1960 (AEV, 4150/8/24). Ils ont notamment pour objet l'orientation professionnelle; l'enseignement secondaire; l'octroi de bourses et prêts d'honneur; l'adaptation des programmes des écoles moyennes à ceux des écoles industrielles inférieures; la création de cours populaires; la création d'un diplôme de maturité commerciale; la création d'une école des métiers; la création d'un collège classique pour les jeunes filles dans le Haut-Valais; l'adaptation des maturités cantonales aux maturités fédérales; la prolongation de la scolarité obligatoire ou encore l'amélioration du statut des maîtres. L'urgence de la modernisation du champ de la scolarité publique, que les opposants à la loi de 1947 pressentaient, est aiguë. Les questions posées démontrent le retard pris dans le cadre de l'organisation de l'école valaisanne et, en particulier, au niveau secondaire et professionnel. L'Evêque lui-même est cité au Parlement pour que les ajustements nécessaires aux temps actuels soient effectués sans attendre (Bulletin du Grand Conseil, juin 1954, p. 179). L'école publique doit se renforcer et se développer. Il faut désormais améliorer les conditions matérielles de l'école primaire en augmentant sa durée et en dédoublant les classes surpeuplées. Il faut développer les écoles secondaires ainsi que les écoles professionnelles et commerciales. Le pays ne peut plus s'en passer.

Un immense chantier législatif est alors ouvert. ${ }^{4}$ Tous les partenaires de l'instruction publique y sont conviés: les associations professionnelles de l'enseignement, de l'économie, du commerce, de l'agriculture, les autorités religieuses, cantonales, communales, l'organisation des femmes catholiques, celle de la jeunesse catholique - entre autres - répondent à l'appel. Ensemble, ils réunissent les informations sur l'état de l'instruction publique dans les autres cantons, ils prennent en compte les commentaires de la presse locale et suisse. Toutes les demandes, motions et postulats déposés au cours des dernières années sont intégrés dans le projet. L'école publique valaisanne, au-delà de l'école primaire, est à créer. La nouvelle loi sur l'instruction publique est donc attendue avec impatience tant par le monde politique que par les représentants des milieux économiques et par le peuple (voir à ce sujet les Bulletins du Grand Conseil de janvier et février 1962. Débats sur la loi sur l'instruction publique).

La démocratisation des études pour les jeunes gens et les jeunes filles est en marche, grâce au système des bourses et prêts d'honneur. La prolongation de la durée de l'année scolaire, y compris dans les zones rurales, devrait permettre à 
tout un chacun d'acquérir le bagage minimal lui permettant de poursuivre sa scolarité dans l'école secondaire communale en particulier. L'engagement des instituteurs est désormais soumis à des règles édictées par l'Etat afin de prévenir les abus; leur salaire est rendu annuel et significativement augmenté, leur permettant enfin de jouir de la considération sociale due à leur fonction (Bulletin du Grand Conseil, juillet 1963, pp. 21-198). Relevons que l'égalité de traitement entre hommes et femmes est en revanche refusée, "parce que cela coûterait trop cher au canton» (p. 107).

La loi sur l'instruction publique de 1962, dont la forme est résolument séculière et ouverte, va susciter l'engouement de tous les partis politiques: le fait est unique dans l'histoire de l'école valaisanne des $19^{\mathrm{e}}$ et $20^{\mathrm{e}}$ siècles. Les socialistes applaudissent à la nouvelle égalité des chances pour tous. Les radicaux se réjouissent de la place donnée à l'enseignement technique et aux formations professionnelles; seul le maintien de l'école confessionnelle suscite leurs regrets sans toutefois remettre en question leur adhésion aux multiples innovations de la loi. Le Parlement en entier se félicite vivement de l'adoption et de la mise en vigueur immédiate de cette "loi du siècle», dont la portée peut effectivement être comparée à celle de la loi de 1873 (à propos de cette loi du $19^{\mathrm{e}}$ siècle, voir Périsset Bagnoud, 1999).

Entre les apports de la loi de 1947 et ceux de la loi de 1962, la distance est immense. L'une tendait à un immobilisme de principe, la seconde introduit plusieurs révolutions. La situation économique du canton a fait évoluer les mentalités à une vitesse inédite. En 1962, une nouvelle page de l'histoire de l'école publique valaisanne commence à s'écrire. La nouvelle législation et les réalisations scolaires qui en sont issues font irrémédiablement basculer le pays dans la modernité. Pourtant, dans l'esprit de la loi, et en contradiction sensible avec les textes, le gouvernement reste attaché à la tradition catholique et rurale, à ses valeurs érigées en dogmes (voir Périsset Bagnoud, 2000). La transmission de cette culture dite valaisanne incombe à l'instituteur, aujourd'hui davantage encore qu'hier, puisque le contexte social n'y souscrit plus entièrement. La réorganisation mesurée de l'Ecole normale évoquée plus haut, réorganisation survenue avant la loi de 1962, devrait permettre la reproduction du système traditionnel auquel tiennent les élites gouvernantes civiles et religieuses.

\section{L'Ecole normale: un chapitre sensible, une idéologie à préserver}

La loi sur l'instruction publique de 1962 contente les défenseurs de la modernité et d'un enseignement mieux ajusté aux nouvelles nécessités économiques. L'attachement religieux demeure cependant inscrit dans la mission de l'école publique: seconder la famille dans l'éducation et l'instruction de la jeunesse, préparer l'enfant à sa tâche d'homme et de chrétien et rechercher à cet effet la collaboration de l'Eglise (Mission générale de l'école, art. 3). Le Parlement a donc admis certains ajustements indispensables, notamment l'accès aux études longues pour les 
classes populaires grâce à la généralisation obligatoire des écoles secondaires et au système des bourses d'études. Mais les assises idéologiques catholiques doivent toujours fonder l'éducation première du peuple, donc l'école primaire. L'internat dans les Ecoles normales reste le moyen privilégié de former les éducateurs chrétiens dont le pays a besoin. L'obligation de l'internat ne saurait être remise en cause malgré les contestations virulentes apparues lors des séances préparatoires de la commission d'experts (AEV, 4150-8/38) puis au Parlement (Bulletin du Grand Conseil, janvier 1962, pp. 122-123). Elle disparait tout au plus des textes de loi. Cependant, le chef du DIP veut toujours des instituteurs et institutrices formés par l'internat: cette exigence, désormais symbolique puisqu'elle a disparu des textes légaux, est néanmoins fermement maintenue. Longtemps encore, les normaliennes et les normaliens continueront à se plier à l'éducation en internat qu'imposent les directions des Ecoles normales quand bien même aucune obligation légale n'y contraint plus.

Afin de renforcer encore l'institution cantonale de formation des maittres, il est décidé par la loi sur l'instruction publique de 1962 d'intituler «Maturité pédagogique» le diplôme d'enseignement délivré par les Ecoles normales. Ce nouveau titre devrait ouvrir les portes des universités de Suisse aux institutrices et instituteurs ${ }^{5}$, accès réservé jusque-là en principe aux seuls étudiantes et étudiants des collèges cantonaux. Autre mesure renforçant les Ecoles normales du canton: l'exclusivité de la formation des institutrices et instituteurs valaisans. Les étudiant(e)s issu(e)s des voies gymnasiales pourront y avoir accès, mais dans des limites permettant de «ne pas ouvrir toutes grandes les voies de l'Ecole normale à ceux qui n'y auront pas reçu la formation complète» (Bulletin du Grand Conseil, févr. 1962, t. 2, p. 148). Pour le Conseil d'Etat, il s'agit bien d'éviter que l'économie, la science pure et l'instruction fonctionnelle prennent le pas sur l'éducation morale et la formation chrétienne. Les Ecoles normales, toujours dirigées par les Congrégations religieuses, en sont garantes. Leur mission est confirmée malgré les innovations consenties: «former de bons instituteurs et des éducateurs chrétiens; donner une formation générale solide et adaptée aux progrès de la science; procurer une formation permettant aux élèves qualifiés de continuer leurs études à l'université» (Rapport de gestion du Conseil d'Etat, DIP, 1962, p. 113). L'économie cantonale reçoit la part d'instruction qui lui revient mais l'éducation morale de la jeunesse valaisanne semble mise à l'abri. L'œuvre politique du gouvernement conservateur tente d'équilibrer les nécessités économiques du pays et le souci idéologique de garantir la pérennité des anciennes valeurs chrétiennes.

Le règlement de 1964 sur les Ecoles normales vient confirmer cette stratégie. Le texte est précis, sobre. La prégnance de l'esprit catholique qui le dirige est formellement éludée. Le gouvernement peut jouer sans danger la carte de la modernité séculière, de l'ouverture. En 1964 toujours, l'Ecole normale des instituteurs emménage dans de nouveaux locaux modernes et spacieux, construits à son intention. Le Gouvernement valaisan les lui a offerts afin de permettre à cette 
formation de se développer de manière à répondre à la demande issue du déploiement des effets de la nouvelle loi sur l'instruction publique. En effet, il faut former de nouveaux instituteurs en nombre: les classes dédoublées réclament davantage de maîtres alors que, dans le même temps, de nombreux régents quittent l'école primaire et vont enseigner dans les nouvelles écoles secondaires communales. Le nouveau directeur de l'Ecole normale, l'abbé Truffer, a les mains libres pour réorganiser le curriculum d'études et le moderniser. Il propose diverses expériences pédagogiques, dont la cogestion, portée aux nues lors de ses débuts en 1967 mais gravement désavouée par le DIP peu d'années plus tard lorsque, les temps ayant changé, elle paraîtra trop s'éloigner des objectifs traditionnels de formation (voir Périsset Bagnoud, 2000).

La loi sur l'instruction publique de 1962, le règlement de 1964 sur les Ecoles normales et l'inauguration du bâtiment de l'Ecole normale des instituteurs en 1964 encore peuvent être considérés comme les points culminants de la politique éducative menée depuis de mitant du $19^{\mathrm{c}}$ siècle par les gouvernements successifs. Mais dès 1970, l'équilibre entre tradition et modernité devient précaire. Pour les Ecoles normales, le temps des turbulences commence.

Avec la bénédiction du gouvernement valaisan, en 1964, l'Ecole normale des instituteurs est donc entrée dans la modernité. L'Ecole normale des institutrices, elle, évolue peu alors, forte de sa formidable confiance en sa mission chrétienne et malgré l'impatience de plus en plus visible des milieux politiques, y compris dans les rangs conservateurs. La divergence des moyens, notamment pédagogiques, engagés par l'une et l'autre Ecole normale fait le lit d'une concurrence délétère entre les deux établissements et facilite le jeu des attaques politiques publiques. En 1969, Sœur Angèle, qui dirigeait l'Ecole normale des institutrices depuis 1935, prend sa retraite. Cette même année, le conseiller d'Etat Marcel Gross, à qui l'on doit la loi sur l'instruction publique de 1962 se retire également. La tradition perd ainsi ses alliés les plus actifs, ses symboles les plus forts. Les réalités $\mathrm{du}$ "monde» (au sens large mais aussi dans le sens que lui attribuent les communautés religieuses, c'est-à-dire ce qui est extérieur à elles), vont, en quelques années bousculer, ébranler, transformer les Ecoles normales.

\section{L'explosion de la demande d'instruction, facteur essentiel du changement}

Alors que l'Ecole normale des instituteurs, à l'aise dans ses nouveaux locaux, peut accueillir un nombre accru de candidats, l'institution responsable de la formation des institutrices effectue sa mue dès 1970. Laccroissement important du nombre de normaliennes force la nouvelle directrice, également religieuse, à revoir les programmes de formation, sans toutefois adopter ceux en vigueur à l'Ecole normale des garçons. En outre, l'effectif désormais élevé d'étudiantes rend impossible la poursuite d'une éducation en internat. Discrètement, en 
1971, la permission est demandée au secrétaire du DIP (et non à son chef) d'en lever l'obligation. La directrice de l'Ecole normale des filles ne renie pas les fondements d'une telle obligation, mais à présent, ce n'est matériellement plus guère possible, d'autant que les parents réclament comme relevant de leur sphère privée l'entière responsabilité de l'éducation de leurs enfants - autre indicateur de la modernité qui a conquis les mentalités valaisannes.

Cependant, malgré ces mesures inédites, la pénurie de personnel enseignant persiste. Il faut recruter des jeunes filles et des jeunes gens issus d'autres écoles générales et les former en quelques mois seulement. Il est ainsi dérogé à la loi séculaire postulant la formation générale, professionnelle mais aussi personnelle du futur instituteur, de la future institutrice, selon les normes attendues par l'Etat. Un cours de formation rapide est organisé en 1963/1964.

\section{L'introduction des cours dits rapides}

Le Valais, ainsi que le relève le Conseil d'Etat dans son rapport, connaît donc lui aussi cette pénurie mondiale du personnel enseignant signalée par le Bureau international d'éducation (BIE) à Genève (Rapport concernant le projet de décision du Conseil d'Etat sur un recrutement complémentaire du personnel enseignant [...]. 26.7.1963. AEV, 4150-7/2). Cause identifiée: profession insuffisamment rémunérée, par conséquent peu attractive. C'est que l'instruction, en ces temps de plein emploi industriel, prend un sens qu'ignoraient auparavant les populations rurales. Les campagnes se dépeuplent. Les villes de plaine accueillent ces nouveaux habitants prêts à travailler dans le secteur public comme dans le privé, d'où une forte demande en matière d'instruction, d'où un besoin accru en personnel enseignant, besoin par ailleurs difficile à satisfaire.

Les normaliens ont bien été appelés à quitter prématurément l'Ecole normale afin de diriger les classes sans maîtres titulaires. Leur effectif, insuffisant, est complété par celui issu du recrutement complémentaire effectué en 1963. Les jeunes filles et jeunes gens admis à cette formation inhabituelle ont été soumis à des conditions strictes afin, espère-t-on, d'éviter des formations au rabais. Les candidat(e)s sont âgé(e)s de 18 ans au minimum. Ils et elles ont déjà acquis un diplôme d'études secondaires (maturité gymnasiale ou diplôme de commerce) ou un certificat de fin d'apprentissage. Sur cinquante, onze candidat(e)s seulement pourront rejoindre le cours dit rapide. Ils et elles sont issu(e)s du collège, d'apprentissages, des écoles d'agriculture ou de commerce. Autre transgression remarquable à la règle traditionnelle: la mixité de la formation. Le DIP suit de près l'évolution de ces futur(e)s enseignant(e)s. Examens d'admission écrits et examens psychotechniques, notes et commentaires sur leurs attitudes professionnelles et personnelles, tout est soigneusement consigné (voir AEV, 4150-8-476). Pour leur plus grande surprise d'ailleurs, les cadres du DIP sont bien obligés de reconnaître la qualité générale de ces étudiant(e)s hors normes. Un nouveau cours de formation rapide est organisé en 1968, puis chaque année dans les deux Ecoles normales jusqu'en 1976, lorsque la pléthore succède à la pénurie. Depuis 
1963, il est donc possible de devenir enseignant(e) sans avoir à se soumettre aux cinq ans de formation normalienne. La tradition est battue en brèche.

Ce nouvel état de fait suscite aussi crainte et méfiance, principalement auprès de quelques membres du clergé particulièrement attachés à la formation globale des futur(e)s instituteur(trice)s. Selon eux, une formation abrégée ne pourrait faire naître un véritable esprit de corps professionnel empreint d'un habitus forgé par un dispositif éducatif adéquat au sortir de l'enfance, le temps d'une brève adolescence. Les cours dits rapides laissent donc devenir instituteurs ou institutrices des jeunes ayant été éduqués non par les Ecoles normales mais par d'autres écoles ayant développé elles-mêmes d'autres cultures appropriées à d'autres missions. C'est ainsi qu'un chanoine, enseignant à l'Ecole normale des filles, déplore l'absence «d'éducation appropriée» et «le manque de vocation» des jeunes filles arrivées dans les dernières classes (lettre du 28.2.1969. AEV, 4150-1987/35 vol. 64). Le directeur de l'Ecole normale lui-même enchérit: il a aussi constaté «l'enracinement moins profond» de ces jeunes filles, leur «volonté d'approfondissement moins accusée» étant donné "qu'il n'est pas toujours possible d'allier la qualité aux impératifs actuels d'une formation massive de candidats à l'enseignement» (lettre du 5.3.1969. AEV, 4150-1992/48 vol. 1). La volonté de voir l'Ecole normale se charger de la globalité de l'éducation des futur(e)s enseignant(e)s des degrés primaires est encore bien présente. Cependant, la conjoncture moderne la met à mal sans qu'aucune volonté politique ne vienne plus au secours des défenseurs de la tradition éducative des Ecoles normales. La situation de crise l'emporte sur toute autre considération, même morale. La formation régulière dispensée par les Ecoles normales donne encore satisfaction, mais les besoins nouveaux imposent des dispositifs inédits. Si l'impact négatif de ces dispositifs novateurs est craint de quelques acteurs, notamment par les enseignants religieux des deux Ecoles normales, leur portée réelle sur la politique de formation des enseignants ne sera mesurée que plus tard. ${ }^{6}$

\section{Une vocation délaissée par les meilleurs: un effet de la démocratisation des études?}

Dans le courant des années '60, le métier d'instituteur n'attire plus les meilleurs élèves des villages. Les candidats sont recrutés en nombre, mais les responsables du DIP s'inquiètent de leur qualité. Il est vrai que, dès 1962, l'école secondaire est généralisée jusque dans les communes éloignées des centres urbains. Dans la foulée, les écoles de commerce régionales offrent leur formation à un public élargi. L'Ecole normale n'est donc plus la seule à instruire après l'école primaire les jeunes issus des milieux ruraux. Le recrutement massif dans les Ecoles normales est peut-être responsable de la baisse de la qualité intellectuelle des futurs institutrices et instituteurs, mais il s'avère aussi que, dans certaines écoles secondaires, les candidates et candidats les plus doués sont détournés de leur projet de formation en Ecole normale et dirigés notamment vers l'école de commerce (lettre du 15.9.1967. AEV, 4150-1987/35 vol. 64). Les études permettant une 
insertion dans l'économie attirent davantage que le modeste métier d'enseignant. La tendance ne faiblit pas dans les années '70 (voir la lettre du Directeur de l'Ecole normale des instituteurs du 16.1.1979. AEV, 4150-1993/38 vol. 19). Le fait est désormais patent: emprunter la voie de l'enseignement primaire n'est pas, ou plus, synonyme d'ascension sociale. Les élèves les plus doués, pour lesquels l'éventail des possibilités de formation supérieure s'est considérablement élargi à l'orée des années '70, ne l'empruntent guère. Une réforme profonde de la formation des enseignant(e)s devient nécessaire, sans cependant que l'on songe déjà à recruter exclusivement des candidats porteurs de diplômes de formations secondaires supérieures.

Les divergences à propos de la formation des enseignants et des moyens à utiliser s'amplifient dans les années ' 70 . Tous les partis politiques, y compris le parti conservateur, fustigent les Ecoles normales. Ainsi, le parti majoritaire se plaint amèrement des innovations qui déstabilisent la tradition de l'Ecole normale des instituteurs. Outre la cogestion, l'introduction des cours d'éducation sexuelle et les nouvelles méthodes d'enseignement du catéchisme sont dénoncées avec virulence (voir l'éditorial du 6. 4. 1973 de Valais Demain, le journal du parti conservateur). Pour leur part, les socialistes réclament la disparition pure et simple de l'institution (voir leur manifeste Ecole unique publié en 1973). La loi de 1962 est une loi moderne: les arguments juridiques que sa stricte application exige fournissent autant d'armes aux opposants à la formation traditionnelle en particulier dès 1977. Elles affaibliront irrémédiablement l'institution jusqu'à obtenir sa fin symbolique (regroupement, mixité et sécularisation de la direction en 1987) et sa fermeture définitive décidée en 1994 (voir Périsset Bagnoud, 2000).

\section{Apparemment vingt ans, deux siècles pourtant}

Une petite vingtaine d'années sépare les années '50 des années ' 70 . Mais, pour la société valaisanne, les idéaux économiques et politiques de deux siècles s'y rencontrent et se succèdent non sans s'affronter, se mélanger, se déchirer. Au passage des années '70, le Valais, acquis au bénéfice matériel de l'industrialisation, voit se transformer à vive allure la mentalité de sa population. Le Vieux-Pays a indiscutablement changé, acceptant tout du $20^{\mathrm{e}}$ siècle moderne, ses contradictions, ses incertitudes, son abondance économique, son impatience et, surtout, l'abandon de son adhésion et de son obéissance aveugle aux valeurs conservatrices. L'individualisme a gagné les âmes acquises désormais à un catholicisme consommé «à la carte», selon l'expression d'Altermatt (1994). Fidèles miroirs de la société, les Ecoles normales et l'instruction publique en général n'échappent pas à ces contradictions lors de la recherche du nouvel équilibre entre aspirations catholiques et ajustements modernes. Une réponse éducative, qui n'est plus celle d'une éducation dévouée au bien social et collectif traditionnel, est à inventer. A présent que la pluralité et la complexité régissent les mentalités, la voie n'est pas ai- 
sée à trouver. La société valaisanne traditionnelle, unie sous la bannière de sa foi catholique, s'incline devant l'irruption de la modernité, de la sécularisation, de l'ère industrielle, de l'éclatement des valeurs et des repères traditionnels, de la délitescence de la culture catholique dans les régions rurales.

Dès 1970, les structures traditionnelles sont donc définitivement bouleversées. Que les congrégations religieuses conservent la direction des Ecoles normales n'y change rien. Il manque désormais, irrémédiablement, un ingrédient essentiel à la réussite de leur action, ingrédient qu'appellent encore les politiciens dans leurs discours mais qui n'est plus désormais qu'un discours d'intention: la loyauté et l'obéissance à une mission commune, à un but social unanimement partagé. Mais la démocratisation des études, l'ouverture d'autres filières de formation aux jeunes filles et jeunes gens et leur espoir fondé de quitter un milieu d'origine trop modeste marginalisent l'action éducative autrefois exclusive des Ecoles normales.

Pour ces dernières, il est urgent de rejoindre la société civile, pluraliste et sécularisée. Elles doivent devenir modernes - de cette modernité qui, ainsi que le relève Hameline (1998, p. 217), «n'est pas différenciable de la sécularisation de la société occidentale» et s'accompagne du "désenchantement à l'égard de la sacralisation chrétienne du monde». Elles ne sont plus au service d'un idéal social univoque, instruments de la reproduction d'une structure éprouvée. Elles doivent à présent tendre vers la nouveauté, l'inédit, l'incertain. Elles ont à établir un nouveau rapport avec la science et ses progrès, puisque, ainsi que l'explicite Le Bouëdec (1998, p. 153), la culture du progrès technique et scientifique prévaut désormais, parallèlement à l'adoption de nouveaux repères éthiques. Dans une atmosphère de sécularisation généralisée, la connaissance devient une valeur essentielle en dehors des références religieuses. Les Ecoles normales tentent donc de perdurer en se fabriquant une nouvelle identité. Pour remplir leur difficile mission elles cherchent désormais à concilier valeurs chrétiennes et société technique et scientifique.

Si, ainsi que l'explique Veyne (1987), l'histoire est impuissante à dégager la moindre loi prédictive, par contre, la reconstruction des conjonctures, la mise en sens et en lien des événements permet d'entrevoir - à défaut de les décrire complètement - les itinéraires probables ayant précédé et accompagné un bouleversement de type structurel. C'est ainsi qu'on peut penser que la fermeture des Ecoles normales du Valais en juin 2000 a sans doute pour origine l'abandon effectif de la tradition et le phénomène de dispersion des valeurs fondatrices de la société catholique valaisanne. En 1962, lorsque la nouvelle loi sur l'instruction publique est votée qui favorisera la démocratisation des études et la multiplication des filières secondaires et des écoles professionnelles, personne n'entrevoira le terme du processus que constitue la fin des Ecoles normales; personne non plus ne la voudra alors délibérément. C'était seulement le processus de modernisation de la société valaisanne qui se dévoilait, comme en passant. 


\section{Notes}

1 L'examen psychotechnique sera introduit à titre expérimental en 1958 et reconduit jusqu'en 1974.

2 L'Ecole normale des institutrices est pour sa part autorisée à continuer à fonctionner selon sa tradition.

3 Cette disposition restera en vigueur jusqu’à la fin des Ecoles normales, en juin 2000.

4 A propos des travaux préparatoires de la loi sur l'instruction publique de 1962, voir AEV $4150-8 / 32$ à $-8 / 47$.

5 Dans les faits, l'accès aux universités par les normaliennes et les normaliens ne sera acquis que plusieurs années plus tard, après de nombreuses négociations bilatérales menées par le DIP valaisan (voir Périsset Bagnoud, 2000).

6 En effet, ces cours rapides, mixtes avant l'heure et fréquentés par des étudiant(e)s issu(e)s de parcours divers, permettent au canton d'aborder par anticipation ce que pourrait être une formation uniquement professionnelle, détachée de la double formation (professionnelle et éducative) des Ecoles normales. Les résultats de la formation dite rapide ont été appréciés par les cadres du DIP. Les désastres annoncés par la Papauté au sujet de la mixité ne se sont pas produits, les enseignant(e)s qui y ont été formés se sont avérés aussi bons que leurs collègues éduqués pendant cinq ans. Lorsqu'il s'agira de réfléchir à une formation essentiellement professionnelle et mixte, dès la fin des années 1980, cette expérience positive permettra aux décideurs de prendre certaines mesures fortes, telles le regroupement des Ecoles normales en 1987 puis la programmation de leur disparition en 1994 (voir Périsset Bagnoud, 2000). Pour le Valais, les cours rapides sont un exemple probant d'une production sociale non voulue, issue d'un dispositif élaboré au demeurant pour reproduire. Les cours rapides n'avaient pas été élaborés pour favoriser l'évolution moderne de l'Ecole normale vers la mixité ni vers des structures essentiellement professionnelles de formation, et pourtant, ils y ont fortement contribué.

\section{Références bibliographiques}

AEV: Archives de l'Etat du Valais, cote 4150.

Altermatt, U. (1994). Le catholicisme au défi de la modernité. Lausanne: Payot.

Arlettaz, G. (1976). Les transformations économiques et le développement du Valais, 18501914. In Groupe valaisan de sciences humaines (Ed.), Développement et mutation du Valais (pp. 9-62). Sion: Groupe valaisan de sciences humaines.

Arlettaz, G. (1979). Les débuts du socialisme. In Groupe valaisan de sciences humaines (Ed.), Histoire de la démocratie en Valais (1798-1914) (pp. 241-254). Sion: Groupe valaisan de sciences humaines.

Clavien, A. (1988). La naissance du parti socialiste valaisan. In Les origines du parti socialiste en Suisse romande (pp. 189-212). Lausanne: Association pour l'étude de l'histoire du mouvement ouvrier.

Crettol, G. (1951). L'autre défense nationale: la terre paysanne. Sion: Fiorina et Pellet.

Criblez, L. \& Hofstetter, R. (Ed.), Périsset Bagnoud, D. (coll.). (2000). Formation des enseignant (e)s. Histoire et réformes actuelles. Berne: Peter Lang.

Hameline, D. (1998). Modernité et archaïsme en pédagogie. In M. Soëtard \& Ch. Jamet (Ed.), Le pédagogue et la modernité (pp. 207-238). Berne: Peter Lang.

Hofstetter, R., Magnin, C., Criblez, L. \& Jenzer, C. (Ed.). (1999). Une école pour la démocratie. Naissance et développement de l'école primaire publique en Suisse, 19 e siècle. Berne: Peter Lang.

Le Bouëdec, G. (1998). Pédagogie moderne et Foi. In M. Soëtard \& C. Jamet (Ed.), Le pédagogue et la modernité (pp. 151-170). Berne: Peter Lang. 
Lustenberger, W. (1997). Les examens pédagogiques des recrues. Coire: Rüegger.

Métrailler, R. (1978). L'école primaire valaisanne durant la deuxième partie du $19 e$ siècle et son processus de popularisation jusquà la veille de la Première Guerre mondiale. Mémoire de licence. Université de Fribourg, Faculté des Lettres.

Nique, C. (1989). Comment l'école devint une affaire d'Etat. Paris: Nathan.

Nique, C. (1991). L'impossible gouvernement des esprits: histoire politique des Ecoles normales primaires. Paris: Nathan.

Périsset Bagnoud, D. (1999). L'instruction primaire publique en Valais (1830-1885). Des législations cantonales à leur application. In R. Hofstetter, C. Magnin, L. Criblez \& C. Jenzer (Ed.), Une école pour la démocratie. Naissance et développement de l'école primaire publique en Suisse au 19e siècle (pp. 137-150). Berne: Peter Lang.

Périsset Bagnoud, D. (2000). Vocation: régent, institutrice. Jeux et enjeux autour des Ecoles normales du Valais romand, 1848-1994. Thèse de doctorat $\mathrm{n}^{\circ} 285$. Université de Genève: Faculté de psychologie et des sciences de l'éducation.

Protocoles des séances du Grand Conseil, 1944-1970. Sion: Bibliothèque cantonale.

Roduit, B. (1993). Les collèges en Valais de 1870 à 1925. Tradition ou modernisation. Lausanne: Société d'histoire de la Suisse romande.

Veyne, P. (1971). Comment on écrit l'histoire. Essai d'épistémologie. Paris: Seuil.

\section{Wenn eine Gesellschaft sich wandelt. Zur Entwicklung der Walliser Lehrerinnen- und Lehrerbildung zwischen 1950 und 1970}

\section{Zusammenfassung}

Der Beitrag analysiert die Bildungspolitik des Kantons Wallis, die den Wandel eines Teils der öffentlichen Schulen zwischen 1950 und 1970 begleitet. Der Einbruch der Moderne und die Demokratisierung des Bildungssystems hatten ausgeprägte Folgen, insbesondere weil der Kanton bis dahin seine industrielle Entwicklung stark gehemmt und an seiner überkommenen politischen und sozialen Tradition festgehalten hatte. Dies änderte sich in den 1960er-Jahren. Das Bildungssystem wurde demokratisiert, die Mittelschulen öffneten sich für breite Bevölkerungsschichten und die Berufe des tertiären Beschäftigungssektors zogen die jungen Leute an. Der Lehrerberuf verlor an Attraktivität. Neue Ausbildungsmodelle wurden eingeführt, welche auch den Niedergang des Lehrerseminars als traditioneller Form der Lehrerinnen- und Lehrerbildung einleiteten.

\section{Quando una società si trasforma. L'evoluzione della formazione degli insegnanti vallesani tra il 1950 e il 1970}

\section{Riassunto}

Questo contributo analizza, dal punto di vista della politica cantonale dell'educazione, una parte delle trasformazioni della scuola pubblica vallesana tra il 1950 e il 1970. Le conseguenze dell'avvento della modernità e della democratizzazione 
degli studi si sono fatte sentire in modo determinante in questo periodo, soprattutto perché fino a quel momento l'evoluzione industriale del cantone era stata molto contenuta e le sue tradizioni rurali, politiche e sociali si erano mantenute pressoché intatte. Gli anni '60 sono forieri di cambiamenti. Alla democratizzazione degli studi si affianca la generalizzazione delle scuole secondarie e una maggiore attrattività degli impieghi nel settore terziario per i giovani. Il mestiere dell'insegnante perde fascino; si inventano nuovi modelli di formazione proprio a partire dalla scomparsa della scuola normale, tradizionalmente deputata alla formazione dei maestri.

\section{When a society changes itself: concerning the development of teacher education in the canton of Wallis between 1950 and 1970}

\section{Summary}

The contribution analyzes the politics of education in the canton of Wallis that accompanied the transformation of parts of the public schools between 1950 and 1970. The advent of modernity and the democratization of the system of education had marked consequences, in particular because so far the canton had strongly inhibited the development of the industry and had held on to its outdated political and social tradition. That changed in the 1960's. The system of education was democratizised, the middle schools opened themselves to broad classes of society, and professions of the tertiary sector of occupations attracted young people. The teaching profession lost some attraction. New models of training were introduced that also initiated the downfall of the teacher's college as the traditional form of teacher training. 
T h e m a 